\title{
PREDITORES DE INSUFICIÊNCIA RENAL CRÔNICA EM PACIENTES DE CENTRO DE REFERÊNCIA EM HIPERTENSÃO ARTERIAL
}

\author{
Cinthia Esbrile Moraes ${ }^{1}$, Camila Farias Cerolli ${ }^{1}$, Vanessa Santos Silva ${ }^{2}$, Roberto Jorge da Silva Franco ${ }^{3}$, Francisco Habermann ${ }^{4}$, \\ Beatriz Bojikian Matsubara ${ }^{5}$, Luis Cuadrado Martin ${ }^{6 *}$ \\ Trabalho realizado na Faculdade de Medicina de Botucatu - UNESP, Botucatu, SP
}

\author{
*Correspondência: \\ Disciplina de Nefrologia, \\ Departamento de Clínica \\ Médica, Faculdade de \\ Medicina de Botucatu, \\ Universidade Estadual \\ Paulista, \\ Rubião Júnior s/nº, Botucatu \\ São Paulo-Brasil. \\ Caixa postal: 584 \\ CEP: 18618-970 \\ Telefone: (014) 38116213; \\ Fax: 014 38822238; \\ cuadrado@fmb.unesp.br
}

\begin{abstract}
RESUMO
OBJEtıvo. Testar variáveis clínicas relacionadas à evolução para insuficiência renal crônica avaliadas rotineiramente em hipertensos e que possam ser utilizadas como instrumento preditivo, possibilitando seu acesso em qualquer nível de assistência.

MÉтodos. Foram avaliados 358 pacientes do Centro de Hipertensão Arterial da Faculdade de Medicina de Botucatu - UNESP. Destes, 210 apresentavam uma segunda avaliação da filtração glomerular e foram utilizados na análise. Aplicou-se regressão logística para identificar características clínicas que se associassem de maneira independente com o desfecho filtração glomerular final igual ou inferior a $60 \mathrm{ml} / \mathrm{min}$.

Resultados. No total da casuística, apenas a proteinúria à urina I associou-se de maneira independente ao desfecho. Entre os 175 pacientes com filtração glomerular inicial superior a $60 \mathrm{ml} / \mathrm{min}$, a presença de proteinúria à urina I, o gênero feminino e a idade igual ou superior a 50 anos foram preditores da evolução para filtração glomerular final igual ou inferior a $60 \mathrm{ml} / \mathrm{min}$.

Conclusão. A proteinúria avaliada à urina I foi um fator de risco associado ao desenvolvimento de insuficiência renal crônica independente de outros co-fatores analisados. Hipertensos primários com proteinúria à urina I devem receber atenção redobrada no sentido de prevenir a evolução para insuficiência renal crônica.
\end{abstract}

UnITERMOS: Hipertensão. Insuficiência renal crônica. Nefroesclerose.

\section{INTRODUÇÃO}

Hipertensão é definida como pressão arterial igual ou superior a $140 \times 90 \mathrm{~mm}$ Hg em adultos com mais de 18 anos, medida em repouso de quinze minutos e confirmada em três aferições consecutivas e em várias visitas médicas ${ }^{1}$. Os rins são órgãoschave na fisiopatologia da hipertensão arterial, sendo que a capacidade de excreção de sódio e a exacerbação da secreção de renina desenvolvem papel primordial na gênese desta patologia².

O aumento do nível pressórico tem sido ligado à doença renal desde 1835, quando Richard Bright descreveu uma doença na qual a necropsia mostrava pacientes com rins contraídos e coração hipertrofiado ${ }^{3}$. Atualmente, a hipertensão é a segunda maior causa de insuficiência renal crônica terminal ${ }^{4,5,6}$. As duas patologias podem estar ligadas principalmente de duas maneiras: a hipertensão arterial, quando em fase maligna, pode levar a quadro de nefroangiosclerose por endarterite obliterante e arteriolite necrotisante; e a forma dita "benigna" pode levar ao quadro de nefrosclerose hipertensiva e perda progressiva e lenta da função renal ${ }^{7}$.

A insuficiência renal crônica terminal (IRCT), quadro em que há necessidade de diálise ou transplante renal, é uma situação clínica cuja prevalência no Brasil é de aproximadamente 391/ 1.000.000 de habitantes ${ }^{8}$. A prevalência e incidência da IRCT têm aumentado progressivamente em escala mundial com crescente aumento nos custos para o sistema de saúde ${ }^{6,10}$.

No Multiple Risk Intervention Trial, aproximadamente 350.000 hipertensos foram seguidos e o risco de desenvolvimento de IRCT foi de 15 / 100.000 hipertensos por ano ${ }^{11}$. Dessa forma, temos que grande parte dos pacientes em diálise chegou a esta situação tendo como causa básica a hipertensão arterial $^{4}$. No entanto, a minoria dos hipertensos desenvolve insuficiência renal. Esse paradoxo deve-se à elevada prevalência da hipertensão arterial na população geral. Assim, mesmo que uma porcentagem pequena dos hipertensos desenvolva IRCT,

1. Sextoanista da Faculdade de Medicina de Botucatu, Botucatu, SP

2. Médica Nefrologista da Faculdade de Medicina de Botucatu, Botucatu, SP

3. Professor adjunto da Faculdade de Medicina de Botucatu, Botucatu, SP

4. Professor Assistente de Nefrologia da Faculdade de Medicina de Botucatu, Botucatu, SP

5. Professora adjunta da Faculdade de Medicina de Botucatu, Botucatu, SP

6. Professor Assistente de Nefrologia - Departamento de Clínica Médica, Faculdade de Medicina de Botucatu, Universidade Estadual Paulista, Botucatu, SP 
o número absoluto desses indivíduos será grande, pois há um risco pequeno aplicado a uma grande parcela da população ${ }^{12}$.

O tratamento da hipertensão arterial é uma das poucas medidas sabidamente efetivas em prolongar a sobrevida renal dos portadores de nefropatia das mais variadas etiologias ${ }^{11,13}$. Quanto maior o grau de proteinúria, mais intensamente a pressão arterial deve ser reduzida ${ }^{9,10}$. Ainda, a utilização de inibidores da enzima conversora e dos antagonistas da angiotensina II tem um efeito protetor renal independente do seu efeito na pressão arterial $\left.\right|^{9,14,15}$.

Com o número crescente de pacientes em diálise é interessante identificar, entre os hipertensos, quais evoluirão para IRCT, uma vez que, dessa maneira, poder-se-á traçar estratégias de proteção renal para reduzir a frequência dessa complicação $0^{9,16,17}$.

Estudo transversal, realizado na Toscana ${ }^{18}$, identificou-se que marcadores genotípicos desfavoráveis relacionados ao sistema renina-angiotensina-aldosterona poderiam estar associados à nefrosclerose hipertensiva. Entretanto, a identificação desses genes não é disponível na atenção primária à saúde. Por outro lado, esses marcadores genéticos estão associados a lesões de órgãos-alvo da hipertensão arterial observáveis à história clínica, exame físico, fundoscopia ou ao eletrocardiograma ${ }^{19}$, variáveis essas que são rotineiramente avaliadas em qualquer nível de atendimento. Não há, na literatura, trabalhos que avaliem marcadores prognósticos para a insuficiência renal crônica em uma coorte de hipertensos brasileira.

Desse modo, o presente estudo teve por objetivo testar variáveis clínicas avaliadas rotineiramente em hipertensos que possam ser utilizadas como instrumento preditivo, acessível em qualquer nível de assistência, relacionadas à evolução para IRCT entre os portadores de hipertensão arterial tratados no Centro de Hipertensão Arterial (CHA) do Hospital das Clínicas da Faculdade de Medicina de Botucatu - UNESP (HC-FMB-UNESP). Assim, poder-se-ia desenvolver estratégias terapêuticas para prevenção desta complicação para o subgrupo de pacientes identificado.

\section{MÉtodos}

Foram avaliados os 358 portadores de hipertensão arterial primária em seguimento no Centro de Hipertensão Arterial ( $\mathrm{CHA})$ do HC-FMB-UNESP, que iniciaram tratamento de janeiro de 1995 até dezembro de 2005. Em nossa instituição os portadores de hipertensão secundária são seguidos em ambulatórios específicos. O presente trabalho obedece à Resolução 196/96 do Conselho Nacional de Saúde e foi aprovado pelo Comitê de Ética desta instituição (processo número: 2323/2006).

Os prontuários desses pacientes foram revistos e os dados clínicos transcritos em protocolo padronizado. Foram analisadas as seguintes variáveis: idade, gênero, raça, altura, peso, índice de massa corpórea, níveis pressóricos, níveis de colesterol, triglicerídeos, HDL-colesterol, creatinina, ácido úrico, presença de proteinúria à urina I, antecedentes de diabetes e tabagismo e medicamentos prescritos nas consultas iniciais e finais.

Anotou-se a presença de lesões de órgãos-alvo (LOA) da hipertensão arterial, definidos a seguir.

Foi considerado sinal de sobrecarga ventricular esquerda o índice de Sokolov (somatória da voltagem da maior onda S em V1 ou V2 com a maior onda R em V5 ou V6) superior a $35 \mathrm{~mm}$.

As alterações em fundo de olho foram estratificadas segundo a presença ou ausência de alterações. Foram calculadas as taxas de filtração glomerular inicial e da última avaliação pela fórmula validada no estudo MDRD (modification of diet in renal disease) com auxílio da tabela publicada por Bastos ${ }^{20}$. A partir desse cálculo, considerando a consulta inicial, a casuística foi analisada como um todo ou estratificada e analisados apenas os pacientes apresentando filtração glomerular inicial maior que $60 \mathrm{ml} / \mathrm{min}$. Em cada grupo foi estabelecido como desfecho filtração glomerular final menor ou igual a $60 \mathrm{ml} / \mathrm{min}$ (grau 3 de insuficiência renal crônica).

Os dados de distribuição normal foram expressos como média \pm desvio padrão. Os dados de distribuição não-paramétrica foram expressos em mediana (primeiro e terceiro quartis). Os pacientes que atingiram o desfecho foram comparados aos que não atingiram pelo teste do Qui quadrado. As variáveis que diferiram com $p<0,1$ foram selecionadas para regressão logística, na qual a associação estatisticamente significante com o desfecho foi considerada ao nível de significância $p<0,05$.

\section{Resultados}

Dos 358 pacientes analisados, apenas variáveis de 210 foram utilizadas nas estatísticas, pois os restantes não possuíam dados para o cálculo da filtração glomerular estimada pela fórmula do MDRD. Na casuística, $61 \%$ eram mulheres, a média de idade foi de $49 \pm 11$ anos com 89\% de brancos. Em relação às comorbidades: $35 \%$ eram tabagistas e $28 \%$ diabéticos. A média de peso foi de 75,6 $\pm 15,5 \mathrm{~kg}$, de altura 1,62 $\pm 0,1 \mathrm{~m}$, resultando em IMC médio de $28,8 \pm 6,3 \mathrm{Kg} / \mathrm{m}^{2}$.

Observou-se redução da pressão arterial ao se comparar a primeira avaliação com a última PA disponível $(p<0,0001)$ : PAS e PAD iniciais de $161 \pm 28,8$ e $103 \pm 17,4$ mm Hg, respectivamente, e PAS e PAD finais de $145 \pm 25,3$ e $90 \pm 12,7 \mathrm{~mm}$ $\mathrm{Hg}$, respectivamente.

Dos exames bioquímicos avaliados, na primeira consulta obteve-se colesterol total médio de $214 \pm 48,6 \mathrm{mg} / \mathrm{dl}$, triglicérides de $141(93,3$ - 195,8) mg/dl, HDL de $40 \pm 12 \mathrm{mg} /$ dl, LDL de $140 \pm 40 \mathrm{mg} / \mathrm{dl}$ e ácido úrico de 5,4 $\pm 2 \mathrm{mg} / \mathrm{dl}$. A creatinina média foi de $1,13 \pm 0,84 \mathrm{mg} / \mathrm{dl}$ e a filtração glomerular de $77,9 \pm 21,72 \mathrm{ml} / \mathrm{min}$. Algum grau de proteinúria à urina I estava presente em 20 de 201 pacientes (10\%), cuja urina I encontrava-se disponível nas consultas iniciais.

Na última consulta, a creatinina média foi de 1,39 $\pm 1,53$ $\mathrm{mg} / \mathrm{dl}$, a filtração glomerular de 74,3 $\pm 25,4 \mathrm{ml} / \mathrm{min}$ e a proteinúria estava presente em 43 de 186 pacientes (23\%) cuja urina I encontrava-se disponível .

Em relação ao exame de fundo de olho, inicialmente, 62\% dos pacientes apresentavam algum grau de alteração hipertensiva (KW grau I no mínimo), evoluindo para 75\% na avaliação final.

Na consulta inicial, $50 \%$ dos pacientes utilizavam diuréticos, $16 \%$ inibidores da enzima conversora, $19 \%$ bloqueadores do canal de cálcio, 13\% beta-bloqueadores, 0,4\% bloqueadores do receptor de angiotensina, 22\% simpatolíticos, 1,4\% vasodilatadores, 3,3\% hipoglicemiantes, 4,8\% ácido acetilsalicílico, $0 \%$ estatinas e 3,4\% alopurinol. Já na consulta final, 57\% dos pacientes utilizavam diuréticos, 63\% inibidores da enzima conversora, $27 \%$ bloqueadores do canal de cálcio, 14\% betabloqueadores, 8,7\% bloqueadores do receptor de angiotensina, $12 \%$ simpatolíticos, $3 \%$ vasodilatadores, $16 \%$ hipoglicemiantes, 16,5\% ácido acetilsalicílico, 13,6\% estatinas. 


\begin{tabular}{|c|c|c|c|}
\hline \multirow[b]{2}{*}{ Variável } & \multicolumn{2}{|c|}{ Desfecho: insuficiência renal } & \multirow[b]{2}{*}{$p$} \\
\hline & Sim & Não & \\
\hline Gênero: masculino / Total (\%) & $13 / 45(29 \%)$ & $68 / 165(41 \%)$ & $>0,1$ \\
\hline Idade: $\geq 50$ anos / Total (\%) & $33 / 45(73 \%)$ & $70 / 165(42 \%)$ & $<0,001$ \\
\hline Raça: negros / Total (\%) & $3 / 45(6 \%)$ & $17 / 162(10 \%)$ & $>0,1$ \\
\hline Tabagismo: fumantes / Total (\%) & $13 / 35(37 \%)$ & $48 / 141(34 \%)$ & $>0,1$ \\
\hline Diabetes: portadores / Total (\%) & $16 / 39(41 \%)$ & $36 / 141(25 \%)$ & $<0,1$ \\
\hline IMC $\geq 30 \mathrm{Kg} / \mathrm{m} 2$ / Total (\%) & $27 / 44(61 \%)$ & $38 / 120(32 \%)$ & $<0,01$ \\
\hline PAS inicial $\geq 160 \mathrm{~mm} \mathrm{Hg} /$ Total & $31 / 45(69 \%)$ & $80 / 165(48 \%)$ & $<0,05$ \\
\hline PAS final $\geq 160 \mathrm{mmHg} /$ Total & $18 / 46(61 \%)$ & $33 / 129(20 \%)$ & $<0,05$ \\
\hline PAD inicial $\geq 110$ mm Hg / Total & $23 / 45(51 \%)$ & $55 / 165(33 \%)$ & $<0,05$ \\
\hline PAD final $\geq 110 \mathrm{mmHg} /$ Total & $3 / 45(6 \%)$ & 18/162 (11\%) & $>0,1$ \\
\hline F0 alterado / Total (\%) & $21 / 26(81 \%)$ & $44 / 77(57 \%)$ & $<0,1$ \\
\hline FG inicial: $<90 \mathrm{ml} / \mathrm{min} /$ Total (\%) & $42 / 44(95 \%)$ & $115 / 166(69 \%)$ & $<0,01$ \\
\hline Proteinúria qualitativa: presente/ Total (\%) & $15 / 42(36 \%)$ & $5 / 159(3 \%)$ & $<0,01$ \\
\hline Colesterol: $\geq 200 \mathrm{mg} / \mathrm{dl} /$ Total (\%) & $29 / 42(69 \%)$ & $91 / 157(58 \%)$ & $>0,1$ \\
\hline Triglicérides: $\geq 150$ mg/dl / Total (\%) & $22 / 41(54 \%)$ & $64 / 146(44 \%)$ & $>0,1$ \\
\hline HDL-colesterol $\leq 40$ mg/dl / Total (\%) & $23 / 31(74 \%)$ & $65 / 113(57 \%)$ & $>0,1$ \\
\hline LDL-colesterol $\geq 130$ mg/dl / Total (\%) & $21 / 31(68 \%)$ & $54 / 100(54 \%)$ & $>0,1$ \\
\hline Ácido úrico $\geq$ 6,0 mg/dl / Total(\%) & $12 / 33(36 \%)$ & $49 / 132(37 \%)$ & $>0,1$ \\
\hline Índice de Sokolov: $\geq 35$ mm/total (\%) & $9 / 45(20 \%)$ & $26 / 163(16 \%)$ & $>0,1$ \\
\hline
\end{tabular}

PAS: pressão arterial sistólica; PAD: pressão arterial diastólica; FO: fundoscopia; FG: filtração glomerular

Tabela 2 - Regressão logística dos fatores preditores de evolução para filtração glomerular inferior ou igual a $60 \mathrm{ml} / \mathrm{min}$ na casuística total de 210 pacientes

\begin{tabular}{lccc}
\hline Variável & S.E. & Significância & Exp $(\beta)$ \\
\hline DM & 0,3852 & 0,8153 & 0 \\
FG inicial $<90 \mathrm{ml} / \mathrm{min}$ & 0,5732 & 0,3793 & 0 \\
Fundo de olho alterado & 0,4062 & 0,5103 & 0 \\
Idade $\geq 50$ anos & 0,3984 & 0,1787 & 0 \\
IMC $\geq 30 \mathrm{Kg} / \mathrm{m} 2$ & 0,3846 & 0,6655 & 0 \\
PAS inicial $\geq 160 \mathrm{~mm} \mathrm{Hg}$ & 0,4350 & 0,3777 & 0 \\
PAS final $\geq 160 \mathrm{~mm} \mathrm{Hg}$ & 0,4191 & 0 & 0 \\
Presença de Proteinúria & 0,4787 & 0,5291 & 1,7089 \\
\hline
\end{tabular}

DM: diabetes melito; FG: filtração glomerular; IMC: índice de massa corpórea; PAS: pressão arterial sistólica

No decorrer da evolução, $21 \%$ evolveram para filtração glomerular $\leq 60 \mathrm{ml} / \mathrm{min}$ (classificado como grau 3 de IRC) e 4,2 \%, para filtração glomerular $\leq 15 \mathrm{ml} / \mathrm{min}$ (grau $5 \mathrm{de} I R C$ ), sendo que, no início do seguimento, de todos os pacientes, $14 \%$ já apresentavam IRC pelo menos grau II. Na presente casuística, $22(10,4 \%)$ evoluíram para óbito em tempo de seguimento médio de 10 anos.

As Tabelas 1 e 2 referem-se à casuística total. Na Tabela 1 observa-se que as variáveis idade $\geq 50$ anos, portadores de diabetes, IMC $\geq 30 \mathrm{~kg} / \mathrm{m}^{2}$, PAS inicial e final $\geq 160 \mathrm{~mm} \mathrm{Hg}$, PAD inicial $\geq 110 \mathrm{~mm} \mathrm{Hg}$, fundo de olho alterado, filtração glomerular inicial $<90 \mathrm{ml} / \mathrm{min}$ e presença de proteinúria associaram-se ao desenvolvimento de insuficiência renal grau 3. A Tabela 2 mostra regressão logística que identificou como fator de risco independente de evolução para filtração glomerular final $\leq 60$ $\mathrm{ml} / \mathrm{min}$ apenas a presença de proteinúria (risco relativo de 3,2).
Nas Tabelas 3 e 4 a amostra foi estratificada e selecionados apenas os pacientes com filtração glomerular inicial $>60 \mathrm{ml} /$ min. Gênero, idade $\geq 50$ anos, filtração glomerular inicial $<90$ $\mathrm{ml} / \mathrm{min}$ e presença de proteinúria associaram-se ao desenvolvimento de insuficiência renal grau 3 (Tabela 3). Na regressão múltipla expressa na Tabela 4, o gênero masculino, idade $\geq 50$ anos e presença de proteinúria associaram-se a evolução para filtração glomerular $\leq 60 \mathrm{ml} / \mathrm{min}$ com risco relativo de 0,41 ; 2,00 e 3,50; respectivamente.

\section{Discussão}

O presente estudo detectou, em coorte de hipertensos, tratados em instituição de ensino médico em centro de referência para o tratamento da hipertensão arterial, uma associação entre presença de proteinúria ao exame de urina I e evolução para deterioração da função renal, independentemente do clearance 
Tabela 3 - Fatores preditores de evolução para filtração glomerular inferior ou igual a $60 \mathrm{ml} / \mathrm{min}$ entre os 175 pacientes com filtração glomerular superior a $60 \mathrm{ml} / \mathrm{min}$

\begin{tabular}{|c|c|c|c|}
\hline \multirow[b]{2}{*}{ Variável } & \multicolumn{2}{|c|}{ Desfecho: insuficiência Renal } & \multirow[b]{2}{*}{$p$} \\
\hline & Sim & Não & \\
\hline Gênero: masculino / Total (\%) & $5 / 25(20 \%)$ & $65 / 150(43 \%)$ & $<0,05$ \\
\hline Idade: $\geq 50$ anos / Total (\%) & $18 / 25(72 \%)$ & $62 / 150(41 \%)$ & $<0,01$ \\
\hline Tabagismo: fumantes / Total (\%) & $4 / 18(22 \%)$ & $44 / 129(34 \%)$ & $>0,1$ \\
\hline Diabetes: portadores / Total (\%) & $9 / 23(39 \%)$ & $32 / 127(25 \%)$ & $>0,1$ \\
\hline IMC $\geq 30 \mathrm{Kg} / \mathrm{m} 2 /$ Total (\%) & $9 / 18(50 \%)$ & $47 / 123(38 \%)$ & $>0,1$ \\
\hline PAD inicial $\geq 110 \mathrm{~mm} \mathrm{Hg} /$ Total & $11 / 25(44 \%)$ & $49 / 150(32 \%)$ & $>0,1$ \\
\hline PAD final $\geq 110 \mathrm{mmHg} /$ Total & $1 / 25(4 \%)$ & $13 / 146(9 \%)$ & $>0,1$ \\
\hline FO alterado / Total (\%) & $10 / 13(77 \%)$ & $45 / 74(61 \%)$ & $>0,1$ \\
\hline FG inicial: $<90 \mathrm{ml} / \mathrm{min} /$ Total (\%) & $17 / 18(94 \%)$ & $105 / 157(67 \%)$ & $<0,05$ \\
\hline Proteinúria qualitativa: presente/ Total (\%) & $4 / 16(25 \%)$ & $6 / 152(4 \%)$ & $<0,01$ \\
\hline Colesterol: $\geq 200 \mathrm{mg} / \mathrm{dl} /$ Total (\%) & $12 / 17(70 \%)$ & $82 / 148(55 \%)$ & $>0,1$ \\
\hline Índice de Sokolov: $\geq 35 \mathrm{~mm} /$ total (\%) & $1 / 19(5 \%)$ & $27 / 156(17 \%)$ & $>0,1$ \\
\hline
\end{tabular}

PAS: pressão arterial sistólica; PAD: pressão arterial diastólica; FO: fundoscopia; FG: filtração glomerular

Tabela 4 - Regressão logística dos fatores preditores de evolução para filtração glomerular inferior ou igual a $60 \mathrm{ml} / \mathrm{min}$ entre os 175 pacientes com filtração glomerular superior a $60 \mathrm{ml} / \mathrm{min}$

\begin{tabular}{|c|c|c|c|c|}
\hline Variável & S.E. & Significância & $\mathbf{R}$ & $\operatorname{Exp}(\beta)$ \\
\hline Idade $\geq 50$ anos & 0,2841 & 0,0145 & 0,1747 & 2,0033 \\
\hline
\end{tabular}

FG: filtração glomerular

inicial, da pressão arterial e de quaisquer outros co-fatores analisados. Essa observação reveste-se de importância, à medida que podemos identificar um subgrupo de pacientes com maior risco de evolução para deterioração renal e envidar esforços para sua prevenção. O valor escolhido para determinação do desfecho foi filtração glomerular $\leq 60 \mathrm{ml} / \mathrm{min}$, pois o risco cardiovascular aumenta marcadamente abaixo desses níveis ${ }^{21}$.

Grandes estudos epidemiológicos realizados em outros países têm identificado como fatores de risco para o desenvolvimento de insuficiência renal crônica em hipertensos a filtração glomerular reduzida, a presença de proteinúria, bem como sua remissão ${ }^{22}$, o diabetes, o tabagismo e os níveis pressóricos ${ }^{23}$.

É assunto controverso se a hipertensão arterial isolada pode causar disfunção renal ou se os hipertensos que evoluem para IRC já apresentavam uma doença renal primária. Estudo recente identificou a elevação da PA como fator de risco independente para o desenvolvimento de insuficiência renal em 316.675 pessoas, cuja integridade renal havia sido documentada previamente, dado que corrobora a idéia de que realmente a nefrosclerose hipertensiva "benigna" possa levar à insuficiência renal. No presente estudo, a observação da maior freqüência de proteinúria na última avaliação disponível em relação à primeira apoia esta ideia ${ }^{24}$. Estudo de nosso meio que avaliou histologicamente pacientes com hipertensão arterial e proteinúria identificou padrões anatomopatológicos compatíveis com nefroesclerose hipertensiva "benigna" ou maligna em dois terços dos casos, por outro lado, um terço destes pacientes apresentava padrão anatomopatológico compatível com nefropatias primárias ${ }^{25}$. Assim, é possível que um terço dos pacientes com proteinúria do presente estudo tivesse na verdade alguma nefropatia primária, porém o significado prognóstico da proteinúria permanece inalterado.

Para os pacientes com filtração glomerular inicial superior a $60 \mathrm{ml} / \mathrm{min}$, as variáveis gênero feminino e idade maior ou igual a 50 anos também se associaram à progressão para insuficiência renal. A disfunção renal avaliada pela fórmula do MDRD, logo no início do seguimento, previu um decréscimo ainda maior da filtração glomerular, ainda que este efeito tenha se atenuado na análise múltipla. Dessa maneira, devemos ficar mais atentos a estes estratos na prevenção da doença renal crônica. 
A frequência de pressão arterial diastólica superior ou igual a $110 \mathrm{~mm} \mathrm{Hg}$ apesar de apresentar diferença estatisticamente significante entre os grupos, não foi incluída na análise múltipla, pois a colinearidade com a pressão arterial sistólica poderia atenuar a significância estatística destas variáveis. Ademais, incluindo-se no modelo a referida variável, não houve alteração significativa do resultado da regressão (dados não apresentados).

Analisando os dados relacionados ao perfil lipídico, os pacientes mostraram tendência à dislipidemia na consulta inicial, a qual, concomitante à hipertensão arterial, compõe critérios para definição de síndrome metabólica. Essa síndrome tem sido identificada na literatura como potente fator de risco para o desenvolvimento de insuficiência renal crônica ${ }^{26}$. Em nosso estudo, por não dispormos da medida da circunferência abdominal, não pudemos identificar os critérios avaliados de síndrome metabólica como preditores de IRC.

Com os dados dos medicamentos utilizados, podemos inferir que, com o passar do tempo, drogas hoje sabidamente efetivas para o controle das comorbidades e dos fatores de risco foram mais utilizadas: estatinas, hipoglicemiantes e aspirina.

A raça negra, que tem maior predisposição à hipertensão arterial e à insuficiência renal, não se mostrou mais propensa ao desfecho avaliado no estudo. Em decorrência da miscigenação frequente no Brasil, é possível que os brancos possuam componentes genéticos da raça negra, o que Ihes confere uma predisposição maior a estas morbidades, minimizando assim as potenciais diferenças.

Alguns prontuários não puderam ser analisados, pois se encontravam inacessíveis pelo arquivo médico do serviço, prejudicando o número de dados obtidos. O número de indivíduos do estudo pode ter abrandado algumas correlações. Ainda assim, obteve-se um número expressivo para apontar os fatores de risco mais relevantes.

Dois fatores de risco para o desenvolvimento de insuficiência renal (presença de doença cardiovascular prévia e tempo de duração da hipertensão arterial) ${ }^{27}$ não foram avaliados no presente trabalho, entretanto, mesmo que estes se associem com o prognóstico renal, isto não anula o fato de que pacientes com proteinúria avançaram mais frequentemente para insuficiência renal e, portanto não impede a validação da proteinúria à urina I como marcador prognóstico.

\section{Conclusão}

Este é o primeiro trabalho brasileiro de nosso conhecimento que avaliou fatores prognósticos para o desenvolvimento de insuficiência renal crônica em uma coorte de hipertensos. 0 presente estudo identificou a proteinúria como marcador prognóstico para declínio da função renal independentemente da filtração glomerular inicial. Isso salienta a importância do exame de urina I, que deve ser realizada independentemente do nível de função renal, na rotina diagnóstica em indivíduos hipertensos como instrumento para predição do desenvolvimento futuro de insuficiência renal.

\section{Agradecimentos}

Este trabalho teve suporte financeiro do CNPQ-PIBIC bolsa de iniciação científica número: 107920/2004-2.

Conflito de interesse: não há

\section{SUMMARY}

\section{Predictors of Chronic Renal failure in PATIENTS of a hyper- TENSION CENTER}

PURPOSE. To verify which clinical variables can predict the evolution to chronic renal insufficiency in routinely evaluated hypertensives.

MetHODS. 358 patients from the Hypertension Center of the Botucatu School of Medicine (São Paulo State University) were evaluated. Sequential evaluation of glomerular filtration rate was detected in 210 patients, who were analyzed. Logistic regression was applied to identify clinical variables independently associated with the development of chronic renal insufficiency with a final glomerular filtration rate equal to or below $60 \mathrm{ml} / \mathrm{min}$.

RESULTS. In routine urinalysis only proteinuria was independently associated with the outcome. Among 175 patients with initial glomerular filtration rate above $60 \mathrm{ml} / \mathrm{min}$, proteinuria, female gender and age of 50 years or more were predictors of the evolution to a final glomerular filtration rate equal to or below $60 \mathrm{ml} / \mathrm{min}$.

CONCLUSION. The presence of proteinuria in simple urinalysis was a risk factor and a reliable predictor associated with the development of chronic renal insufficiency among hypertensives. [Rev Assoc Med Bras 2009; 55(3): 257-62]

KEY WORDS: Hypertension. Chronic renal insufficiency. Nephrosclerosis.

\section{REFERÊNCIAS}

1. Kaplan NM. Hypertension in the population at large. In: Kaplan NM. editor. Clinical hypertension 7th ed. Baltimore: Williams \& Wilkins; 1998. p:1-19.

2. Kaplan NM. Primary hypertension: pathogenesis. In: Kaplan NM, editor. Clinical hypertension. 7th ed. Blatimore: Williams \& Wilkins; 1998. p.41-101.

3. Eknoyan G. On the central role of studies on the kidney in the recognition, conceptual evolution, and understanding of hypertension. Adv Chronic Kidney Dis. 2004; 11:192-6.

4. Mazzuchi N, Schwedt E, Fernandez JM, Cusumano AM, Ancao MS, Poblete $\mathrm{H}$, et al. Latin American Registry of dialysis and renal transplantation: 1993 annual dialysis data report. Nephrol Dial Transplant. 1997;12:2521-7.

5. Held PJ, Port FK, Webb RL, Wolfe RA, Garcia JR, Blagg CR,et al. The United States Renal Data Systems 1991 annual data report: an introduction. Am J Kidney Dis. 1991;18:1-16

6. Coresh J, Astor BC, Greene T, Eknoyan G, Levey AS. Prevalence of chronic kidney disease and decreased kidney function in the adult US population: Third National Health and Nutrition Examination Survey. Am J Kidney Dis. 2003;41:1 -12.

7. Kaplan NM. Renal parenchymal hypertension. In: Kaplan NM, editor. Clinical hypertension. 7th ed. Baltimore: Williams \& Wilkins; 1998. p.281-301.

8. Censos. [citado $18 \mathrm{fev}$ 2008]. Disponíveis em: http://www.sbn.org.br/ Censo/2007/SBN Censo Diálise 2007.doc.

9. Hebert LA, Wilmer WA, Falkeñhain ME, Ladson-Wofford SE, Nahman Jr. NS, Rovin BH. Renoprotection: One or many Therapies? Kidney Int. 2001;59:1221-26

10. Norris KC, Greene T, Kopple JJ, Lea J, Lewis J, Lipkowitz M, et al for the AASK Study Group. Baseline predictors of renal disease progression in the African American Study of Hypertension and Kidney Disease. J Am Soc Nephrol.2006;17:2928-936.

11. Klag MJ, Whelton PK, Randall BL, Neaton JD, Brancati FL, Ford CE, et al. Blood pressure and end-stage renal disease in men. N Engl J Med. 1996;334:13-8.

12. Chobanian AV, Bakris GL, Black HR, Cushman WC, Green LA, Izzo JL Jr, et al. National Heart, Lung, and Blood Institute Joint National Committee on Prevention, Detection, Evaluation, and Treatment of High Blood Pressure. National High Blood Pressure Education Program Coordinating Committee. The Seventh Report of the Joint National Committee on Prevention, Detection, Evaluation, and Treatment of High Blood Pressure: the JNC 7 report. JAMA. 2003;289:2560-72.

13. Shulman NB, Ford CE, Hall WD, Blaufox MD, Simon D, et al. Prognostic value of serum creatinine and effect of treatment of hypertension on renal function. Results from the hypertension detection and follow-up program. 
The Hypertension Detection and Follow-up Program Cooperative Group. Hypertension. 1989;13:180-93.

14. Agodoa LY, Appel L, Bakris GL, Berck G, Bourgoignie J, Briggs JP, et al. African American Study of Kidney Disease and Hypertension (AASK) Study Group. Effect of ramipril vs amlodipine on renal outcomes in hypertensive nephrosclerosis: a randomized controlled trial. JAMA. 2001;285:2719-28

15. Gruppo Italiano di Studi Epidemiologici in Nefrologia (GISEN). Randomised placebo-controlled trial of effect of ramipril on decline in glomerular filtration rate and risk of terminal renal failure in proteinuric, non-diabetic nephropathy. Lancet 1997;349:1857-63.

16. Palmer BF. Renal dysfunction complicating the treatment of hypertension. $\mathrm{N}$ Engl J Med. 2002; 347:1256-61.

17. Pedrini MT, Levey AS, Lau J, Calmers TC, Wang PH. The effect of dietary protein restriction on the progression of diabetic and nondiabetic renal diseases: a meta-analysis. Ann Intern Med. 1996;124:627-32.

18. Fabris B, Bortoletto M, Candido R, Barbone F, Cattin MR, Calci M, et al. Genetic polymorphisms of the renin-angiotensin-aldosterone system and renal insufficiency in essential hypertension. J Hypertens. 2005;23:309-16.

19. Lindpainter K, Pfeffer MA, Kreutz R, Stampfer MJ, Grodsteini F, et al. A prospective evaluation of an angiotensin-converting-enzyme gene polymorfism and the risk of ischemic heart disease. N Engl J Med. 1995;332:706-11.

20. Bastos MG. Método prático para avaliação da filtração glomerular. J Bras Nefrol. 2006;38:21-4

21. Foster MC, Hwang S, Larson MG, Parikh NI, Meigs JB, Vasan RS, et al. Cross-classification of microalbuminuria and reduced glomerular filtration rate: associations between cardiovascular disease risk factors and clinical outcomes. Arch Intern Med. 2007;167:1386-92

22. Lea J, Greene T, Hebert L, Lipkowitz M, Massry S, Middleton J, et al. The relationship between magnitude of proteinuria reduction and risk of end-stage renal disease: results of the African American study of kidney disease and hypertension. Arch Intern Med. 2005;165:947 -53.

23. Haroun MK, Jaar BG, Hoffman SC, Comstock GW, Klag MJ, Coresh J: Risk factors for chronic kidney disease: A prospective study of 23,534 men and women in Washington County, Maryland. J Am Soc Nephrol. 2003;14:2934 -941.

24. Hsu C, McCulloch CE, Darbinian J, Go AS, Iribarren C. Elevated blood pressure and risk of end-stage renal disease in subjects without baseline kidney disease. Arch Intern Med. 2005;165:923-8.

25. Caetano ERSP, Zatz R, Saldanha LB, Praxedes JN. Hypertensivenephrosclerosis as a relevant cause of chronic renal failure. Hypertension. 2001;38:171-6.

26. Peralta CA, Kurella M, Lo JC, Chertow GM. The metabolic syndrome and chronic kidney disease. Curr Opin Nephrol Hypertens. 2006;15:361-5.

27. Marín R, Gorostidi M, Fernández-Vega F, Alvarez-Navascués R. Systemic and glomerular hypertension and progression of chronic renal disease: the dilemma of nephrosclerosis. Kidney Int Suppl. 2005;99:52-6.

Artigo recebido: 10/03/08 Aceito para publicação: 13/09/08 